DOI 10. 18307/2015. 0613

(C) 2015 by Journal of Lake Sciences

\title{
过氧化钙在处理厌氧底泥中的应用初探"
}

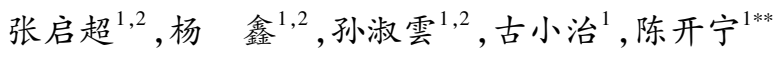 \\ ( $1:$ 中国科学院南京地理与湖泊研究所湖泊与环境国家重点实验室,南京 210008) \\ (2: 中国科学院大学, 北京 100049$)$
}

\begin{abstract}
摘 要: 为改善河道厌氧底质及内源氮、磷等营养盐释放问题, 考察对沉水植被恢复的影响, 研发可同步解决沉积物供氧和 削减内源氮、磷释放的氧缓释材料. 实验通过向沉积物一水界面处散点注射不同剂量的过氧化钙 $\left(\mathrm{CaO}_{2}\right)$, 研究界面处溶解氧 的动态变化特征及表层沉积物与底层水体之间溶解态氮、磷的交换过程. 结果表明: 添加 $\mathrm{CaO}_{2}$ 显著提高了界面处底层上覆 水溶解氧浓度, 随着 $\mathrm{CaO}_{2}$ 浓度的增加溶解氧浓度增加, 不同处理组之间具有显著差异; $\mathrm{CaO}_{2}$ 对沉积物中 $\mathrm{PO}_{4}^{3-}-\mathrm{P}$ 释放具有 明显的抑制作用, 且随 $\mathrm{CaO}_{2}$ 浓度的增加抑制效果愈加明显, 上覆水中可溶性活性磷浓度最大可削减 $98 \%$. 实验开始时, 磷释 放速率可降至 $-241.916 \pm 22.501 \mathrm{mg} /\left(\mathrm{m}^{2} \cdot \mathrm{d}\right)$, 降幅最高可达到 $144 \% ; \mathrm{CaO}_{2}$ 对沉积物 $\mathrm{NH}_{4}^{+}-\mathrm{N}$ 释放的抑制效果不佳, 上覆 水中 $\mathrm{NH}_{4}^{+}-\mathrm{N}$ 浓度随着时间的变化波动性较大, 且有逐渐增大的趋势. 另外, 添加 $\mathrm{CaO}_{2}$ 会显著提高底层上覆水 $\mathrm{pH}$ 值, 不同处理 组之间差异显著, 但当 $\mathrm{CaO}_{2}$ 投加量小于 $0.529 \mathrm{~kg} / \mathrm{m}^{2}$ 时, 不会对苦草种子的萌发生长有显著影响, $\mathrm{pH}$ 值波动在可接受范围内 (7.62 10.87). 因此, 结合污染沉积物的状况, 适当地投加 $\mathrm{CaO}_{2}$ 有望同步解决底质厌氧、内源磷释放及后期沉水植被定植底质生 境改善的问题,可推荐为一种黑臭污染底泥治理技术在实际的河道生态工程中应用,其适宜浓度为 $0.176 \mathrm{~kg} / \mathrm{m}^{2}$ 左右.
\end{abstract}

关键词: 黑臭河道; 厌氧底质;过氧化钻; 上覆水; $\mathrm{pH}$ 值; 溶解氧; 氮磷释放

\section{Preliminary study about application of calcium peroxide in the heavily contaminated sedi- ments remediation}

\author{
ZHANG Qichao ${ }^{1,2}$, YANG Xin ${ }^{1,2}$, SUN Shuyun ${ }^{1,2}$, GU Xiaozhi ${ }^{1}$ \& CHEN Kaining ${ }^{1}$ \\ (1: State Key Laboratory of Lake Science and Environment, Nanjing Institute of Geography and Limnology, Chinese Academy \\ of Sciences, Nanjing 210008, P. R. China) \\ (2: University of Chinese Academy of Sciences, Beijing 100049, P. R. China)
}

\begin{abstract}
In order to rehabilitate the anaerobic sediment in Moon River, a controllable indoor experiment was carried out. It aimed at finding a synchronization solution of providing oxygen for the anaerobic sediment, reducing the endogenous nutrients release rate, as well as building up plant communities during the later recovery. In the controllable experiment, different concentrations of $\mathrm{CaO}_{2}$ was injected to the surface of sediment separately. It suggested that adding $\mathrm{CaO}_{2}$ could significantly improve the concentrations of dissolved oxygen in the lying water, even more it had a positive correlation with the changes of $\mathrm{CaO}_{2}$ concentration. It had a significantly negative impact on the release of $\mathrm{PO}_{4}^{3-}-\mathrm{P}$ in the sediment, more significant with the increasing concentrations of $\mathrm{CaO}$. The concentration of $\mathrm{PO}_{4}^{3-}-\mathrm{P}$ could be decreased by $98 \%$ mostly in the overlying water contrasted with the control group. The release rate of $\mathrm{PO}_{4}^{3-}-\mathrm{P}$ could be decreased from $-99.069 \pm 55.991 \mathrm{mg} /\left(\mathrm{m}^{2} \cdot \mathrm{d}\right)$ to $-241.916 \pm 22.501 \mathrm{mg} /\left(\mathrm{m}{ }^{2} \cdot \mathrm{d}\right)$. On the other hand, the concentration of $\mathrm{NH}_{4}^{+}-\mathrm{N}$ in the overlying water rose with fluctuations. It had significantly improved the $\mathrm{pH}$ in the overlying water. But it had little impact on the growth of Vallisneria natans when the concentrations of $\mathrm{CaO}_{2}$ was lower than $0.529 \mathrm{~kg} / \mathrm{m}^{2}$. Considering the pollution status, adding $\mathrm{CaO}_{2}$ could reduce the release of $\mathrm{PO}_{4}^{3-}-\mathrm{P}$ and enhance the anaerobic sediment suited for submerged macrophytes growth. Consequently, adding $\mathrm{CaO}_{2}$ could be routinely performed to solve heavily contaminated sediments in the actual river ecological engineering, with the proper dosage of $0.176 \mathrm{~kg} / \mathrm{m}^{2}$.
\end{abstract}

* 太湖水污染治理专项 ( TH2013213, TH2013310) 和中国科学院重点部署项目 ( KZZD-EW-10-05) 联合资助. 2014-09-22收稿;2015-03-11 收修改稿. 张启超(1991～), 男, 硕士研究生; E-mail: njzhangqichao@ 163. com.

** 通信作者;E-mail:knchen@ niglas. ac. cn. 
Keywords : Black and odorous water; anaerobic sediment; calcium peroxide; lying water; $\mathrm{pH}$; dissolved oxygen ; release of nitrogen and phosphorus

随着我国环境污染治理及管理力度的增强, 河道的外源性污染物得到大幅度削减. 但河道的内源性污 染物仍会导致水质恶化, 难以改善. 底泥作为污染物的聚集体, 是最大的内源性污染源 ${ }^{[1-5]}$. 疏浚是解决底泥 污染的有效措施之一, 然而考虑到施工条件、成本、效果等因素, 并不是所有受污染河道都适合采用该措 施 $^{[6]}$. 因此, 前期可采用化学修复治理水环境, 同时也可以作为应急处置手段改善基底环境, 易于后期生态 修复工作的开展.

溶解氧浓度是评价水体受污染程度和自净能力的重要指标 ${ }^{[7]}$, 水体污染越严重, 溶解氧浓度越低, 更易 引起水土界面处内源磷的释放 ${ }^{[8]}$. 与其他修复试剂相比, 过氧化钲 $\left(\mathrm{CaO}_{2}\right)$ 不仅能控制沉积物中内源磷释放, 而且其作为一种释氧化合物 ( oxygen release compounds, ORC) 能与水反应并缓慢地释放氧气 ${ }^{[9-11]}$, 改善厌氧 底泥性质, 具有供氧快速、操作简单、环境副作用小的特点. 另外, Arienzo 等研究表明, $\mathrm{CaO}_{2}$ 能够极大地促进 微生物增长, 经常作为微生物激活剂来修复水体或者土壤的有机污染物 ${ }^{[10,12]}$. 目前, $\mathrm{CaO}_{2}$ 对水体修复技术的 研究处于起步阶段,具有较好的应用前景.

本文主要利用 $\mathrm{CaO}_{2}$ 作为氧缓释剂来提高沉积物一水界面处上覆水中溶解氧浓度, 以期达到改善表层厌 氧底泥性质、抑制内源性营养盐释放的效果; 在实验室条件下探索对厌氧底泥处理效果较好的 $\mathrm{CaO}_{2}$ 投加剂 量, 为利用 $\mathrm{CaO}_{2}$ 修复厌氧基质提供科学依据.

\section{1 材料和方法}

\section{1 实验材料}

沉积物采集: 用自制柱状采泥器于样点处 $\left(30^{\circ} 45^{\prime} 27.57^{\prime \prime} \mathrm{N}, 120^{\circ} 44^{\prime} 14.85^{\prime \prime} \mathrm{E}\right)$ 采集泥样, 有机玻璃管规格 为内径 $\varnothing 8.5 \mathrm{~cm} \times 60 \mathrm{~cm}$. 沉积物高度不少于 $20 \mathrm{~cm}$, 沉积物上部注满水后两端用橡皮塞塞紧垂直放置, 小心 带回实验室备用. 沉积物的含水率为 $45.22 \% \pm 2.49 \%$, 有机质含量为 $13.02 \% \pm 1.96 \%$, 总氮 $(\mathrm{TN}) 、$ 总磷 (TP) 含量分别为 $4.388 \pm 0.978 、 1.612 \pm 0.026 \mathrm{mg} / \mathrm{g}$.

水样采集:在样点处用 $25 \mathrm{~L}$ 塑料桶采集水样 2 次, 密封遮光保存, 冷藏备用. 水样的 $\mathrm{pH}$ 值为 $7.22 \pm$ $0.06, \mathrm{TN} 、 \mathrm{TP}$ 、铵态氮 $\left(\mathrm{NH}_{4}^{+}-\mathrm{N}\right)$ 、磷酸盐 $\left(\mathrm{PO}_{4}^{3-}-\mathrm{P}\right)$ 浓度和高锰酸盐指数 $\left(\mathrm{COD}_{\mathrm{Mn}}\right)$ 分别为 $13.385 \pm 1.508$ 、 $0.917 \pm 0.077 、 6.299 \pm 0.125 、 0.804 \pm 0.002$ 和 $11.54 \pm 2.20 \mathrm{mg} / \mathrm{L}$.

\section{2 实验设计}

首先抽去有机玻璃管中的上覆水, 再采用虹吸法沿壁缓慢加人用 $0.45 \mu \mathrm{m}$ 醋酸纤维膜过滤后的原位点 水样至距底泥表面 $20 \mathrm{~cm}$ 处, 标记出水面位置. 有机玻璃管编号分别为 $\mathrm{CK} 、 1^{\#} 、 2^{\#} 、 3^{\#} 、 4^{\#}, \mathrm{CK}$ 为空白对照组, $1^{\#} \sim 4^{\#}$ 处理组中分别均匀注人 $0.5 、 1 、 3 、 5 \mathrm{~g} \mathrm{CaO}$ 的水浊液至底泥表面, 每点相互间隔 $2 \mathrm{~cm}$. 此时, 4 个处理组 有机玻璃管中 $\mathrm{CaO}_{2}$ 浓度分别为 $0.088 、 0.176 、 0.529$ 和 $0.881 \mathrm{~kg} / \mathrm{m}^{2}$. 用黑布罩住有机玻璃管, 蔽光培养. 每 种处理重复 3 次. 室内模拟实验从 2014 年 5 月 23 日到 31 日,为期 1 周.

上覆水采集: 实验开始即刻采集已过滤的水样作为初始值, 此后于第 $6 、 12 、 24 、 36 、 60 、 120 \mathrm{~h}$ 采用虹吸法 于上覆水中段取 $50 \mathrm{ml}$ 水样, 同时补充水样至标记处. 所取水样经 $0.45 \mu \mathrm{m}$ 玻璃纤维膜过滤后分析上覆水 $\mathrm{NH}_{4}^{+}-\mathrm{N} 、 \mathrm{PO}_{4}^{3-}-\mathrm{P}$ 浓度. 取样结束后用微电极测定沉积物一水界面处溶解氧 ( DO) 浓度, 分析其垂直变化特征.

\section{3 样品测定与分析}

水体营养盐测定参照《水和废水监测分析方法》 ${ }^{[13]} \cdot \mathrm{NH}_{4}^{+}-\mathrm{N}$ 浓度测定采用纳氏试剂比色法; $\mathrm{TP} \mathrm{PO}_{4}^{3-}-\mathrm{P}$ 浓度测定采用钼钩抗分光光度法; TN 浓度测定采用过硫酸钾消解后紫外分光光度法; 沉积物有机质在马弗 炉中于 $550^{\circ} \mathrm{C}$ 下灼烧 $4 \mathrm{~h}$ 后测定; 沉积物含水率在 $105^{\circ} \mathrm{C}$ 下烘至恒重计算; $\mathrm{pH}$ 值采用 Mettler Toledo FE20 实 验 $\mathrm{pH}$ 计测定; 沉积物一水分界处 DO 浓度分布采用丹麦 Unisense 公司微电极测量系统测定.

\section{4 数据处理}

数据处理分析与绘图采用 Excel 2013 和 Origin 9.0 软件, 沉积物营养盐释放速率计算公式为 ${ }^{[14]}$ :

$$
r=\left[V\left(C_{\mathrm{n}}-C_{0}\right)+\sum_{n-1}^{n} V_{j-1}\left(C_{j-1}-C_{\mathrm{a}}\right)\right] / A \cdot t
$$


式中, $r$ 为释放速率 $\left(\mathrm{mg} /\left(\mathrm{m}^{2} \cdot \mathrm{d}\right)\right), V$ 为柱中上覆 水体积 $(\mathrm{L}), C_{n} 、 C_{0} 、 C_{j-1}$ 分别为第 $n$ 次、初始和 $j-1$ 次采样时的营养盐浓度 $(\mathrm{mg} / \mathrm{L}), C_{\mathrm{a}}$ 为添加水中该 营养盐浓度 $(\mathrm{mg} / \mathrm{L}), V_{j-1}$ 为第 $j-1$ 次采样的体积 $(\mathrm{L}), A$ 为沉积物一水界面的接触面积 $\left(\mathrm{m}^{2}\right), t$ 为释 放时间 (d).

\section{2 结果与分析}

\section{1 静态培养过程中上覆水 $\mathrm{pH}$ 值随时间的变化 曲线}

实验过程中 $\mathrm{CaO}_{2}$ 与水反应生成具有碱性的氢 氧化钲 $\left(\mathrm{Ca}(\mathrm{OH})_{2}\right)$, 导致上覆水 $\mathrm{pH}$ 值升高. 聚集 在沉积物表面的 $\mathrm{Ca}(\mathrm{OH})_{2}$ 向表层扩散使上覆水 $\mathrm{pH}$ 值逐步升高, 随后逐渐趋于稳定达到平衡状态. 实 验结束时 $\mathrm{CK} 、 1^{\#} 、 2^{\#} 、 3^{\#} 、 4^{\#}$ 处理组中 $\mathrm{pH}$ 值分别为 $7.62 \pm 0.02 、 8.61 \pm 0.02 、 9.72 \pm 0.02 、 10.79 \pm$ $0.01 、 10.87 \pm 0.05$, 不同处理组间存在显著差异 $(P<0.05)$ ( 图 1 ).

\section{2 静态培养后 $\mathrm{CaO}_{2}$ 浓度对沉积物一水界面溶解}

\section{氧浓度变化的影响}

在静态培养结束后, 将沉积物柱样静置 $24 \mathrm{~h}$, 利用溶解氧微电极测定分析沉积物一水分界处 DO 浓度的垂直分布特征(图 2), 其中 $0 \mathrm{~mm}$ 处为沉积 物一水分界面, 下方为沉积物. 添加不同浓度 $\mathrm{CaO}_{2}$ 后, 所有处理组沉积物界面处上覆水中 DO 浓度与 对照组相比均有不同程度的提高, 且各处理组间存 在显著差异 $(P<0.05)$. 一般情况下, DO 浓度随着 $\mathrm{CaO}_{2}$ 浓度的升高而增加, 但 $3^{\#}$ 和 $4^{\#}$ 处理组存在异 常, $\mathrm{CaO}_{2}$ 投加浓度较低的 $3^{\#}$ 处理组界面处上覆水 DO 浓度反而更高. 在所有处理组中, 随着 $\mathrm{CaO}_{2}$ 浓 度的增加, DO 更容易渗透到深层间隙水中, 改善 深层基质厌氧环境.

当 $\mathrm{CaO}_{2}$ 投加浓度为 $0.176 \mathrm{~kg} / \mathrm{m}^{2}$ 时, $2^{\#}$ 处理组 中沉积物界面上覆水 DO 浓度为 $7.88 \pm$ $0.06 \mathrm{mg} / \mathrm{L}$, 略高于此温度下水中饱和 DO 浓度 $(7.56 \mathrm{mg} / \mathrm{L})$. 此外, $2^{\#}$ 处理组沉积物间隙水中 DO 浓度变化趋势与对照组一致, 说明此时并无剩余 $\mathrm{CaO}_{2}$ 富集在沉积物表层间隙水中. 当 $\mathrm{CaO}_{2}$ 投加浓 度超过 $0.529 \mathrm{~kg} / \mathrm{m}^{2}$ 时, 在维持上覆水高 DO 浓度 的同时,仍有剩余 DO 富集于 $5 \mathrm{~mm}$ 的表层沉积物间 隙水中, 最高浓度可达到 $30.33 \mathrm{mg} / \mathrm{L}$, 远远超过此 时水中饱和 $\mathrm{DO}$ 浓度. 在测定高浓度 $\mathrm{CaO}_{2}$ 处理组

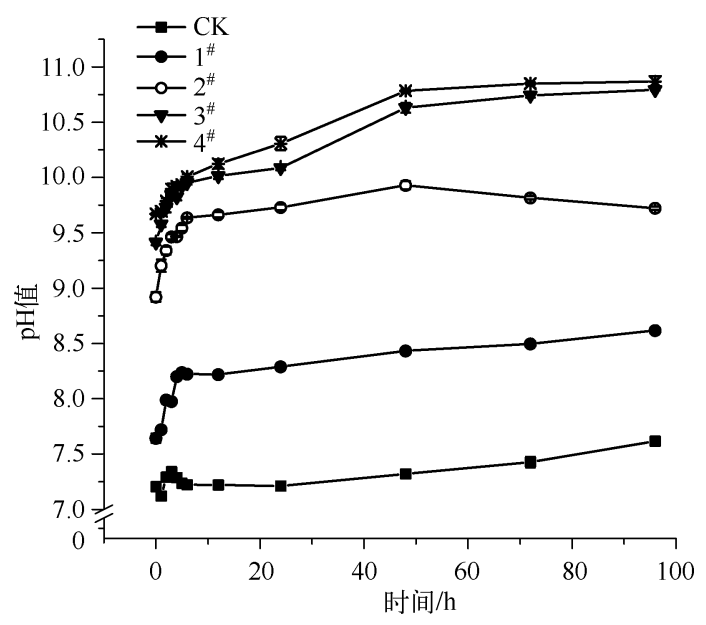

图 1 不同浓度 $\mathrm{CaO}_{2}$ 处理后 $\mathrm{pH}$ 值随时间的变化

Fig. 1 Changes of $\mathrm{pH}$ with time under different concentrations of $\mathrm{CaO}_{2}$

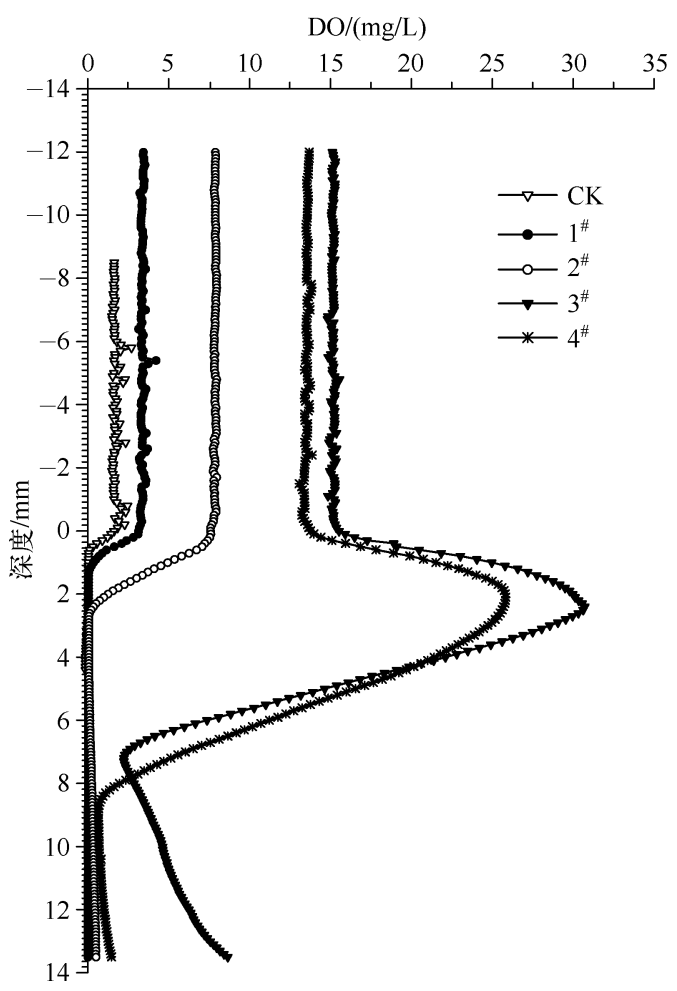

图 2 不同浓度 $\mathrm{CaO}_{2}$ 处理后溶解氧浓度的垂直分布

Fig. 2 Vertical profiles for dissolved oxygen concentration under different concentrations of $\mathrm{CaO}_{2}$ 沉积物间隙水中 DO 浓度时发现, 当 DO 浓度下降到最小值后会有一定幅度的上升, 造成 DO 浓度与实际情 况存在差异. 这可能与测定过程中表层间隙水中 DO 浓度随针状探头孔隙扩散到深层底泥有关. 


\section{3 静态培养过程中 $\mathrm{CaO}_{2}$ 浓度对 $\mathrm{PO}_{4}^{3-}-\mathrm{P}$ 释放的影响}

$\mathrm{PO}_{4}^{3-}-\mathrm{P}$ 浓度及释放速率的变化曲线表明 (图 3), $\mathrm{CaO}_{2}$ 对沉积物界面处内源磷释放的影响主要以抑制为 主, $\mathrm{CaO}_{2}$ 浓度增加时抑制效果更明显. 实验开始阶段, 添加 $\mathrm{CaO}_{2}$ 处理组中 $\mathrm{PO}_{4}^{3-}-\mathrm{P}$ 浓度先下降, 而后随着时间 的推移逐渐趋于平衡, 而对照组中 $\mathrm{PO}_{4}^{3-}-\mathrm{P}$ 浓度先下降后逐渐升高. $\mathrm{PO}_{4}^{3-}-\mathrm{P}$ 浓度的下降幅度随 $\mathrm{CaO}_{2}$ 浓度的 增加而增大, 上覆水中活性磷浓度最大可削减 $98 \%$. 实验结束时, 对照组中 $\mathrm{PO}_{4}^{3-}-\mathrm{P}$ 浓度为 $1.0686 \pm 0.1044$ $\mathrm{mg} / \mathrm{L}$, 与其他处理组之间存在显著性差异 $(P<0.05) .2^{\#}$ 处理组中 $\mathrm{PO}_{4}^{3-}-\mathrm{P}$ 浓度为 $0.3576 \pm 0.0175 \mathrm{mg} / \mathrm{L}$, 与 $1^{\#}$ 处理组差异不显著 $(P>0.05)$, 与 $3^{\#}$ 处理组存在显著差异 $(P<0.05) .4^{\#}$ 处理组中 $\mathrm{PO}_{4}^{3-}-\mathrm{P}$ 浓度为 $0.0171 \pm$ $0.0061 \mathrm{mg} / \mathrm{L}$, 与 $3^{\#}$ 处理组差异不显著 $(P>0.05)$.
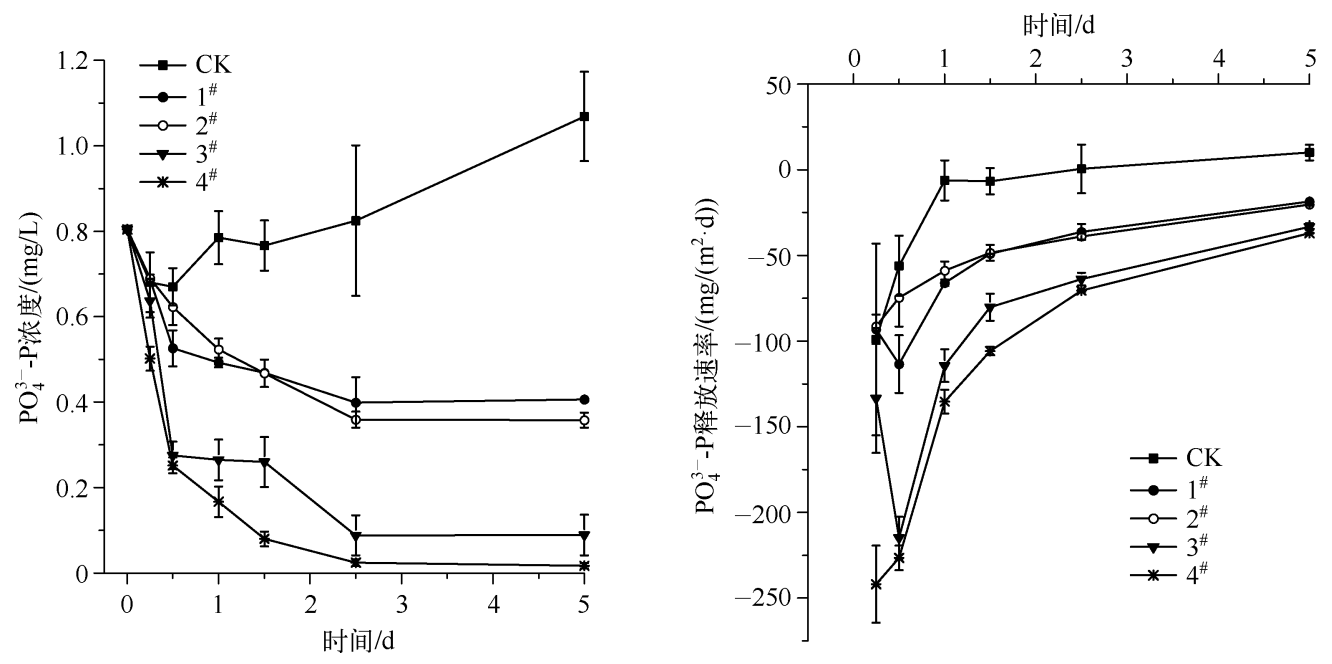

图 3 不同浓度 $\mathrm{CaO}_{2}$ 对 $\mathrm{PO}_{4}^{3-}-\mathrm{P}$ 浓度变化及释放速率的影响

Fig. 3 Changes of concentration and release rate of $\mathrm{PO}_{4}^{3-}-\mathrm{P}$ in the sediment under different concentrations of $\mathrm{CaO}_{2}$

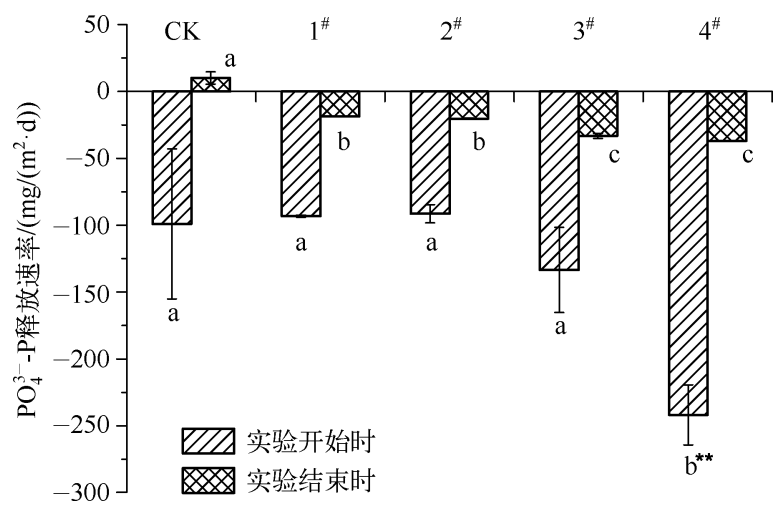

图 4 实验开始、结束时 $\mathrm{PO}_{4}^{3-}-\mathrm{P}$ 的释放速率

Fig. $4 \mathrm{PO}_{4}^{3-}-\mathrm{P}$ release rate of the sediment at the beginning and end of the experiment

在实验开始阶段, $\mathrm{PO}_{4}^{3-}-\mathrm{P}$ 的释放速率只 有 $4^{\#}$ 处理组与其他处理组之间存在显著性 差异 $(P<0.05)$, 说明刚投加到底泥表面的 $\mathrm{CaO}_{2}$ 对降低活性磷浓度迅速产生影响, 并且 当 $\mathrm{CaO}_{2}$ 浓度达到一定浓度时, 磷释放速率才 会出现显著性差异 (图 4). 在实验结束阶 段, 对照组以促进内源释放为主, 与 $\mathrm{CaO}_{2}$ 处 理组之间存在显著性差异 $(P<0.05)$. 同时 所有 $\mathrm{CaO}_{2}$ 处理组中 $\mathrm{PO}_{4}^{3-}-\mathrm{P}$ 释放速率之间的 差异性与 $\mathrm{PO}_{4}^{3-}-\mathrm{P}$ 浓度的差异性基本一致.

\section{4 静态培养过程中 $\mathrm{CaO}_{2}$ 浓度对 $\mathrm{NH}_{4}^{+}-\mathrm{N}$}

\section{释放的影响}

$\mathrm{NH}_{4}^{+}-\mathrm{N}$ 浓度及释放速率的变化曲线表 明 (图 5 ), 在整个实验阶段, 所有处理组中 $\mathrm{NH}_{4}^{+}-\mathrm{N}$ 浓度呈不规则的波动变化, 但是总体呈上升趋势. 投加 $\mathrm{CaO}_{2}$ 对沉积物一水界面处 $\mathrm{NH}_{4}^{+}-\mathrm{N}$ 释放的影响 同样以抑制作用为主, 但不同浓度 $\mathrm{CaO}_{2}$ 处理组之间 $\mathrm{NH}_{4}^{+}-\mathrm{N}$ 浓度差异性不显著 $(P>0.05)$. 在所选浓度范围 内, $\mathrm{CaO}_{2}$ 对水体中 $\mathrm{NH}_{4}^{+}-\mathrm{N}$ 的去除效果不佳. 实验结束阶段, 空白对照组中 $\mathrm{NH}_{4}^{+}-\mathrm{N}$ 浓度为 9.1220 0.5512 $\mathrm{mg} / \mathrm{L}$, 这与浓度较高的 $\mathrm{CaO}_{2}$ 处理组之间存在显著差异 $(P<0.05)$, 与 $1^{\#}$ 处理组差异不显著 $(P>0.05), \mathrm{CaO}_{2}$ 浓度较高的 $2^{\#} 、 3^{\#} 、 4^{\#}$ 处理组之间差异不显著 $(P>0.05)$. 实验结束阶段, $\mathrm{CaO}_{2}$ 处理组中的 $\mathrm{NH}_{4}^{+}-\mathrm{N}$ 释放速率 
小于对照组,差异情况与上覆水中 $\mathrm{NH}_{4}^{+}-\mathrm{N}$ 浓度差异性一致.
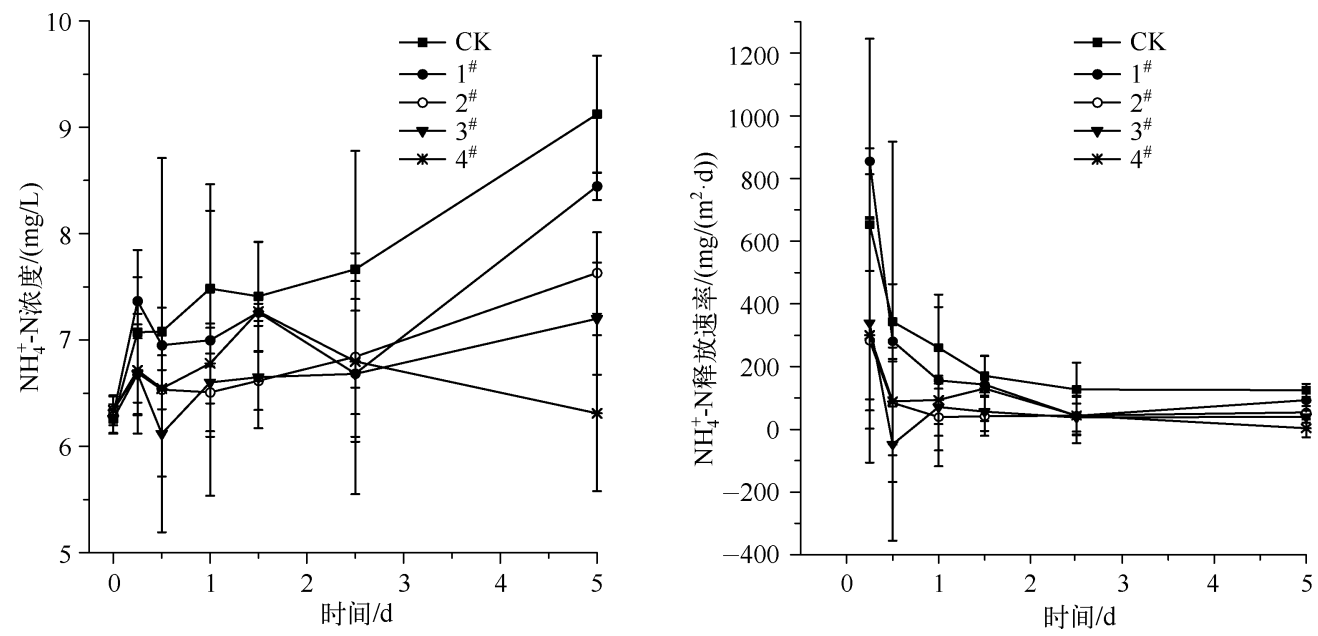

图 5 不同浓度 $\mathrm{CaO}_{2}$ 处理后对 $\mathrm{NH}_{4}^{+}-\mathrm{N}$ 浓度及释放速率的影响

Fig. 5 Changes of concentration and release rate of $\mathrm{NH}_{4}^{+}-\mathrm{N}$ in the sediment under different concentrations of $\mathrm{CaO}_{2}$

\section{3 讨论}

\section{3. $1 \mathrm{CaO}_{2}$ 浓度对沉积物一水界面处 $\mathrm{pH}$ 值变化的影响}

投加 $\mathrm{CaO}_{2}$ 后, 沉积物一水界面处上覆水中 $\mathrm{pH}$ 值明显升高. $\mathrm{CaO}_{2}$ 浓度较高的 $3^{\#}$ 、 $4^{\#}$ 处理组中, 沉积物一水 界面上覆水呈较强的碱性环境, 可能会对原有的水体生态系统造成负面影响. 在水生植被中, 苦草 (Vallisneria natans (Lour.) Hara) 具有繁殖快、再生能力强、破坏后恢复时间短、抗逆性强、耐寒等特点, 能够在碱性较 强的环境中生长良好. 研究表明, 在 CK、1 $1^{\#} 、 2^{\#} 、 3^{\#}$ 处理组中的苦草种子处于萌发状态, 且生长良好, 尤其在 $2^{\#}$ 处理组中苦草生长更为旺盛. 因此, 投加 $\mathrm{CaO}_{2}$ 可能会对原有的生态系统产生影响, 但不会对后期植被修复措 施造成破坏性影响. 因此, $\mathrm{CaO}_{2}$ 可以作为一种化学试剂修复水体环境, 且对水生态环境影响较小.

\section{$3.2 \mathrm{CaO}_{2}$ 浓度对沉积物一水界面处 DO 浓度的影响}

投加不同浓度 $\mathrm{CaO}_{2}$ 后, 所有处理组中沉积物界面处上覆水中的 DO 浓度均不同程度地提高, 并且各处 理组之间存在显著性差异. 这说明投加 $\mathrm{CaO}_{2}$ 作为一种应急措施, 对提高沉积物界面处上覆水 DO 浓度水平 具有明显效果. 因此, 针对 DO 浓度较低的黑臭河道, 可以将 $\mathrm{CaO}_{2}$ 作为前期增氧技术应用到实际工程中. 根 据实验数据分析, 为了以最小的 $\mathrm{CaO}_{2}$ 投加剂量使上覆水 $\mathrm{DO}$ 浓度维持在适宜水平, 降低工程成本, 适宜投加 $0.176 \mathrm{~kg} / \mathrm{m}^{2}$ 的 $\mathrm{CaO}_{2}$.

虽然 $\mathrm{CaO}_{2}$ 是一种缓释氧化合物, 但在实验过程中仍有较高的反应速率, 使底层上覆水 DO 浓度迅速达 到饱和. 实际应用工程中需要将产生的氧气缓慢地释放到水体中, 并维持较长的时间, 减少溶解氧逸散. 因 此, 通过对 $\mathrm{CaO}_{2}$ 进行改性, 既能不改变其抑制磷释放能力的同时, 又可以降低释放氧气的速率, 增强其应用 性. 有部分学者进行过有关 $\mathrm{CaO}_{2}$ 缓释氧材料制作的研究, 如 Bianchi-Mosquera 等 ${ }^{[15]}$ 将释氧剂与水泥混合制 成渗透性较好的混凝土颗粒, 以降低其反应速率; 刘涉江等 ${ }^{[16]}$ 采用混合法将 $\mathrm{CaO}_{2}$ 载人到磷酸钙骨水泥中制 成缓释氧负荷材料改善其释氧速率与释氧周期; 周彦波等 ${ }^{[17]}$ 将 $\mathrm{CaO}_{2}$ 粉末包裹在硬脂肪酸中, 减少 $\mathrm{CaO}_{2}$ 与 水的接触面积, 从而达到降低释氧速率的目的.

\section{$3.3 \mathrm{CaO}_{2}$ 浓度对内源性氮、磷释放的影响}

$\mathrm{CaO}_{2}$ 对控制沉积物中 $\mathrm{PO}_{4}^{3-}-\mathrm{P}^{3} \mathrm{NH}_{4}^{+}-\mathrm{N}$ 释放的效果有明显差异, 投加 $\mathrm{CaO}_{2}$ 明显抑制沉积物中 $\mathrm{PO}_{4}^{3-}-\mathrm{P}$ 的 释放, 但对 $\mathrm{NH}_{4}^{+}-\mathrm{N}$ 释放的抑制效果不佳, 使上覆水中 $\mathrm{NH}_{4}^{+}-\mathrm{N}$ 浓度有小幅度增加. 这可能与 $\mathrm{CaO}_{2}$ 的性质有 关, 上覆水中可溶性活性磷与 $\mathrm{CaO}_{2}$ 反应形成稳定的钙结合态磷, 沉积在底质中, 抑制沉积物中磷释放, 从而 
减少上覆水中可溶性活性磷浓度. 通过实验证明, 在短时间内, 投加 $\mathrm{CaO}_{2}$ 可以一定程度上降低沉积物中 $\mathrm{NH}_{4}^{+}-\mathrm{N}$ 的释放速率.

因此, 投加 $\mathrm{CaO}_{2}$ 主要是通过将水体中的可溶性活性磷转化为稳定的钲结合态磷, 从而达到抑制磷释 放、净化水质的效果. 因此, 针对含有高浓度可溶性活性磷的水体, 通过投加 $\mathrm{CaO}_{2}$ 能起到一定的改善效果.

\section{4 结论}

1) 通过室内培养试验, 投加 $\mathrm{CaO}_{2}$ 可同步解决沉积物界面处厌氧现状和抑制沉积物内源磷释放问题. 对比 该缓释材料在控制内源性氮、磷释放的效果发现, 其在控制磷释放方面效果更佳, 适当增加 $\mathrm{CaO}_{2}$ 投加量, 控制 效果显著提高. 尽管实验中造成局部水体 $\mathrm{pH}$ 值升高,适当控制 $\mathrm{CaO}_{2}$ 投加量不会显著影响苦草种子的萌发.

2) 添加 $\mathrm{CaO}_{2}$ 显著性改变了表层沉积物的 DO 分布, 表层沉积物作为 DO 的源, 不断向底层水体及邻近 底泥释氧, 降低沉积物中磷释放速率. 同时添加 $\mathrm{CaO}_{2}$ 通过增加沉积物活性钙来源实现其固磷作用.

3) $\mathrm{CaO}_{2}$ 对黑臭河道底泥改善的室内模拟实验初步取得成效, $\mathrm{CaO}_{2}$ 作为缓释材料有望在相关的河道生 态工程治理中应用. 当 $\mathrm{CaO}_{2}$ 投加浓度为 $0.176 \mathrm{~kg} / \mathrm{m}^{2}$ 时, 既达到相应的处理效果, 又降低工程成本, 为适宜 投加剂量.

致谢: 采样及实验过程中得到杨拿、孙淑雲的帮助, 在此表示诚挚的谢意.

\section{5 参考文献}

[ 1 ] Zoumis T, Schmidt A, Grigorova L et al. Contaminants in sediments : remobilisation and demobilisation. Science of the Total Environment, 2001, 266(1) : 195-202.

[ 2 ] Ogiwara K, Morgi K. The purification of Lake Suwa (dredging). Proceedings of 6th International Conference on the Conservation \& Management of Lakes-Kasumigaura, 1995.

[ 3 ] Jiang JG, Shen YF. Estimation of the natural purification rate of a eutrophic lake after pollutant removal. Ecological Engineering, 2006, 28(2): 166-173.

[4] 许列峰,陈 婕, 邵之剑. 城市河流生物修复技术的研究进展. 安徽农业科学,2013,41(6):2597-2600.

[5] 赵 振. 氧化试剂 (硝酸钲) 控制黑臭底泥营养盐释放的效果研究. 环境科技,2010,23(4):17-19.

[6] 兴培民,王国祥, 胡春华等. 底泥疏浚能控制湖泊富营养化吗? 湖泊科学, 2000,12(3):269-279. DOI 10. 18307/ 2000.0312.

[ 7 ] 冯海艳, 李文霞, 杨忠芳等. 上覆水溶解氧水平对苏州城市河道底泥吸附、释放磷影响的研究. 地学前缘, 2008, 15 (5) :227-234.

[ 8 ] Kamiya H, Ishitobi Y, Inoue T et al. Effluxes of dissolved organic phosphorus ( DOP) and phosphate from the sediment to the overlying water at high temperature and low dissolved oxygen concentration conditions in an eutrophic brackish lake. Japanese Journal of Limnology ( Japan), 2001, 62 (1):11-21.

[9] 张亚雷, 章 明, 李建华等. $\mathrm{CaO}_{2}$ 不同投加方式对底泥磷释放的抑制效果分析. 环境科学, 2006, 27 (11): 2188-2193.

[10] Arienzo M. Degradation of 2,4,6-trinitrotoluene in water and soil slurry utilizing a calcium peroxide compound. Chemosphere, $2000, \mathbf{4 0}(4): 331-337$.

[11] Gallizia I, Vezzulli L, Fabiano M. Oxygen supply for biostimulation of enzymatic activity in organic-rich marine ecosystems. Soil Biology and Biochemistry, 2004, 36(10): 1645-1652.

[12] White DM, Irvine RL, Woolard CR. The use of solid peroxides to stimulate growth of aerobic microbes in tundra. Journal of Hazardous Materials, 1998, 57 (1) : 71-78.

[13] 国家环境保护总局《水和废水监测分析方法》编委会. 水和废水监测分析方法. 北京: 中国环境科学出版社,2002.

[14] 范成新, 秦伯强, 孙 越. 梅梁湖和五里湖水一沉积物界面的物质交换. 湖泊科学, 1998, 10 (1):73-78. DOI 10. $18307 / 1998.0112$.

[15] Bianchi-Mosquera GC, Allen-King RM, Mackay DM. Enhanced degradation of dissolved benzene and toluene using a solid oxygen-releasing compound. Ground Water Monitoring \& Remediation, 1994, 14(1) : 120-128.

[16] 刘涉江, 刘秀丽, 王少博. $\mathrm{CPC} / \mathrm{CaO}_{2}$ 氧缓释复合材料的制备及其释氧性能探讨. 环境工程学报, 2010,4 ( 5 ) : $971-974$.

[17] 周彦波, 王英秀, 周振华等. 过氧化钙缓释氧剂的制备及其释氧特性研究. 中国给水排水,2012,28(7):64-67. 